\title{
Meldepflichtige Tierseuchen diagnostiziert am Institut für Veterinärpathologie der Universität Zürich zwischen 1988 und 2004
}

\author{
S. Kimpfler ${ }^{1}$, A. Pospischil1, P. Rüsch² ${ }^{2}$ T. Sydler ${ }^{1}$ \\ ${ }^{1}$ Institut für Veterinärpathologie der Universität Zürich, ${ }^{2 K a n t o n a l e s ~ V e t e r i n a ̈ r a m t, ~ Z u ̈ r i c h ~}$
}

\section{Zusammenfassung}

Die Untersuchung und Befunderhebung an Tierkörpern auf makroskopischer und histologischer Ebene ist eine der zentralen Aufgaben der Veterinärpathologie. Häufig wird vom Einsender die Klärung der Krankheits- beziehungsweise Todesursache verlangt ohne einen genauen Vorbericht zu übermitteln oder spezifische Fragen, zum Beispiel nach dem Vorliegen einer Tierseuche, zu stellen. Die Untersuchung gliedert sich in Sektion nach allgemeinem Schema und einer flexiblen Palette von Zusatzuntersuchungen, um kostenbewusst und mit vertretbarem Aufwand den Fall zu klären und die Fragen des Einsenders zu beantworten. Ziel dieser retrospektiven Analyse eines Sektionsgutes von 36365 Tierkörpern und 9192 Organen zwischen 1988 und 2004 war es, Anzahl und Art von Tierseuchen in der Routinediagnostik aufzuklären. In 2918 Fällen (6.4\% aller Untersuchungen) fanden sich meldepflichtige Tierseuchen nämlich bei 2426 Nutztieren ( $9.1 \%$ der Nutztiere) und bei 492 Tieren anderer Spezies $(2.6 \%$ der übrigen Tierarten). Mit diesen gewonnen Daten lassen sich ein erheblicher Nutzen pathologischanatomischer Abklärungen erkennen und bestehende Datenbanken ergänzen.

Schlüsselwörter: Tierseuche, Pathologie, Untersuchungsgang, Schweiz

\section{Einleitung}

Die Tiergesundheit spielt unter gesellschaftlichen und wirtschaftlichen Gesichtspunkten eine bedeutende Rolle. Insbesondere können Tierseuchen den freien Handel von Tieren und Tiererzeugnissen nachhaltig beeinträchtigen. Verschiedene staatliche Institutionen und überstaatliche Organisationen sind in die Bekämpfung und Überwachung von Tierseuchen eingebunden. Weltweit dokumentiert das internationale Tierseuchenamt in Paris (OIE) unter anderem das globale Tierseuchen- und Zoonosegeschehen, erarbeitet Standards für die Tierseuchenbekämpfung und

\section{Notifiable animal diseases diagnosed at the Institute of Veterinary Pathology of the University of Zurich between 1988 and 2004}

One of the essential tasks of veterinary pathology is the gross and microscopic examination of animals post mortem. Frequently requests are made for the cause of disease or death in the absence of a precise history or an otherwise specific assignment e.g. whether a notifiable disease is involved. The general examination is supplemented by a spectrum of additional examinations depending on the case whereas attempts are made to keep the costs within limits and to answer the client's request with justifiable effort. 36365 necropsy cases and 9192 organs submitted between 1988 and 2004 were analysed to give indications on the number and type of notifiable diseases that were diagnosed in the course of routine diagnostic procedures. Notifiable animal diseases were discovered in 2918 cases $(6.4 \%$ of all investigated cases) namely 2426 farm animals $(9.1 \%$ of all farm animals) and 492 animals of other species $(2.6 \%$ of all other animal species). These data illustrate the considerable value of pathological-anatomical examinations and compliment the figures from other databases.

Keywords: notifiable animal disease, pathology, examination, Switzerland

fördert die internationale Zusammenarbeit bei der Tierseuchenkontrolle (OIE Objectives, 2007). Die Europäische Union sieht unter anderem ihre Aufgabe im Schutz und derVerbesserung von Gesundheit und Lebensbedingungen, insbesondere derjenigen Tiere, die der Nahrungsmittelproduktion dienen. Dabei besteht der Grundsatz der Gewährleistung des freien innergemeinschaftlichen Handels mit Tieren und tierischen Erzeugnissen und deren Einfuhr nach Gesundheitsnormen und internationalen Verpflichtungen (Europäische Union, 2007). Eine staatliche 
Bekämpfung von übertragbaren Tierseuchen ist in der Regel immer dann erforderlich, wenn diese derVolkswirtschaft schwere wirtschaftliche Schäden zufügen, wenn sich Tierbesitzer nicht mit eigenen Mitteln ausreichend dagegen schützen können oder wenn sie direkt über Kontakte oder indirekt über vom Tier stammende Lebensmittel die Gesundheit des Menschen bedrohen (Mayr, 2002).

Das eidgenössische Tierseuchengesetz vom 1. Juli 1966 erläutert den Begriff Tierseuchen folgendermassen: "Tierseuchen im Sinne des vorliegenden Gesetzes sind die übertragbaren Krankheiten, die a) auf den Menschen übertragen werden können (Zoonosen), b) vom einzelnen Tierhalter ohne Einbezug weiterer Tierbestände nicht mit Aussicht auf Erfolg abgewehrt werden können, c) einheimische, wildlebende Tierarten bedrohen können, d) bedeutsame wirtschaftliche Folgen haben können, e) für den internationalen Handel mit Tieren und tierischen Produkten von Bedeutung sind" (Art. 1 Abs. 1 TSG). Im gleichen Artikel des eidgenössischen Tierseuchengesetzes wird zwischen hochansteckenden, auszurottenden, zu bekämpfenden und zu überwachenden Seuchen unterschieden (Art. 1 Abs. 2 TSG).

Hochansteckende Seuchen sind Krankheiten mit dem Potenzial sich in grossem Masse und schnell auch über Landesgrenzen hinaus zu verbreiten. Sie haben umfangreiche gesundheitspolizeiliche Konsequenzen und sind von höchster Bedeutung für den internationalen Handel mit Tieren und tierischen Produkten (BVET, 2007). Sie sind nach alter OIE Klassifikation von 2005 in der Liste A der meldepflichtigen Krankheiten aufgeführt (OIE, 2005). Seit den letzten Ausbrüchen der Newcastle Disease 1998, der Maul- und Klauenseuche 1980 und der Klassischen Schweinepest 1993 ist die Schweiz frei von allen hochansteckenden Seuchen. Diese sind grösstenteils noch nie oder schon lange nicht mehr aufgetreten (BVET Bulletin, 03/2007). Bei den im Frühjahr 2006 in der Schweiz nachgewiesenen Fällen von Geflügelpest bei Wildvögeln (HPAI) handelte es sich ausschliesslich um Einzelfälle.Auszurottende Seuchen werden mit finanziell aufwändigen Programmen bekämpft, wodurch gewisse Seuchen in den letzten Jahrzehnten in der Schweiz eliminiert werden konnten oder kurz vor der Tilgung stehen (IBR, BSE, CAE,Tollwut). Bei den zu bekämpfenden Seuchen steht die Schadensbegrenzung im Vordergrund, da der Aufwand einer Ausrottung in keinem sinnvollen wirtschaftlichen Verhältnis zum Nutzen steht. Wichtige Erkrankungen in dieser Gruppe sind die Salmonellose, Coxiellose und die Enzootische Pneumonie (EP) sowie die Aktinobazillose der Schweine (APP). Für zu überwachende Seuchen ist allein eine Meldepflicht vorgeschrieben. Die gesammelten Daten über diese Seuchen sollen im Fall einer eventuellen Bekämpfung die Überführung in eine andere Tierseuchenklasse erleichtern (BVET,
2007). Zu dieser Kategorie gehört zum Beispiel die Bovine Virus Diarrhoe (BVD), deren Ausrottung in der Schweiz ab Herbst 2008 geplant und deshalb eine Umklassierung (von zu überwachende / in auszurottende Tierseuche) vorgesehen ist (BVET Magazin 4-5, 2006).

Die Aufgabe von veterinärpathologischen Institutionen beziehungsweise Laboratorien einschliesslich der universitären Institute ist die Erstellung einer Diagnose auf der Basis einer pathologisch-anatomischen Untersuchung. Diese umfasst die makroskopische Befunderhebung im Rahmen einer Sektion, den Entscheid zur Durchführung unmittelbarer zusätzlicher Laboruntersuchungen und eine Sicherstellung von Gewebeproben für die histologischen oder andere später durchzuführende Untersuchungen. Mit dem Auftrag zur Abklärung der Krankheits- beziehungsweise Todesursache erwartet der Einsender eine breit gefächerte Untersuchung unter Einsatz verschiedenster Methoden. Die histologische Untersuchung ist hierbei die weitaus am häufigsten durchgeführte $\mathrm{Zu}$ satzuntersuchung.

Aus Kostengründen wird der Umfang von Zusatzuntersuchungen auf der Basis einer makroskopischen Verdachtsdiagnose eingeleitet, die wesentlich durch die Erfahrung des untersuchenden Pathologen beeinflusst wird. Zusätzlich schränken Tierart und Alter des Tieres die Differentialdiagnosemöglichkeiten ein. Bereits das Vorliegen eines morphologisch begründeten Verdachts auf eine meldepflichtige Tierseuche verpflichtet dazu, dem zuständigen Kantonstierarzt Meldung zu erstatten beziehungsweise einen Auftrag für die Weitersendung der Proben einzuholen (Art. 11 TSG und Art. 61 TSV). Proben zur Abklärung auf Tierseuchen werden an akkreditierte Unterauftragnehmer weitergeleitet (z.B. bakteriologische Abortuntersuchungen), die über das notwendige Methodenspektrum verfügen. Der Fall wird zusammen mit den Zusatzuntersuchungen vom Pathologen abgeschlossen. In dieser retrospektiven Untersuchung sollen die Funktion und die Möglichkeiten der Pathologie im Rahmen von Diagnose und Ausschluss von Tierseuchen bewertet werden.

\section{Material und Methoden}

\section{Die Datenbank des Instituts für Veterinärpathologie Zürich (IVPZ)}

Die Ergebnisse der Untersuchungen, die am IVPZ durchgefuihrt worden sind (Tab. 1), werden seit 1988 in einer elektronischen Datenbank erfasst und als pathologisch-anatomische Diagnosen beziehungsweise Verdachtsdiagnosen und ätiologische Diagnosen mittels Deskriptoren eingegeben. Tierseuchenfälle beziehungsweise Verdachtsfälle konnten über diese De- 
skriptoren direkt oder indirekt in der Datenbank gefunden werden. In nicht eindeutigen Fällen wurden die Details aus den archivierten Fallprotokollen entnommen. Gezählt wurden alle gefundenen Tierseuchen ab 1988, auch wenn diverse Erkrankungen erst später vom Gesetzgeber zu Tierseuchen erklärt wurden. Im zufällig herausgegriffenen Jahr 2003 wurden Art und Häufigkeit der weiterführenden Untersuchungen und auch Fälle gezählt, bei welchen die Krankheitsursache nicht näher bestimmt werden konnte. Eine detaillierte Analyse der Abortfälle beim Rind wurde für die Jahre 2000 bis 2004 durchgeführt, da seit dieser Zeit Neosporose und BVD regelmässig abgeklärt werden.

\section{Der pathologisch-anatomische Untersuchungsgang und weiterführende Untersuchungen}

Sektionen werden am IVPZ bei landwirtschaftlichen Nutztieren aller Altersstufen und bei verschiedensten Haus-, Heim- Wild- und Zootieren durchgeführt. Die Fragestellung nach der Todes- oder Krankheitsursache ist der häufigste Grund für einen Auftrag zur pathologisch-anatomischen Untersuchung. Dabei wird vom Einsender in der Regel eine umfassende Untersuchung erwartet. An Tierkörpern und Organen werden anhand von Läsionen auf verschiedenen Ebenen (Makroskopie, Histologie) weitere Untersuchungen eingeleitet. Diese diagnostischen Abklärungen, mit Ausnahme von Abortuntersuchungen, sind im Wesentlichen von der Anamnese, den makroskopischen Befunden, der Kosten-/Nutzenabwägung weiterer Laboruntersuchungen und auch von der Erfahrung des Obduzenten abhängig. In einer abschliessenden Bewertung aller Befunde wird versucht, eine Erklärung für die Todes- beziehungsweise Krankheitsursache $\mathrm{zu}$ finden.

\section{Ergebnisse}

\section{Untersuchungen, Tierarten und Tïerseuchen}

Zwischen 1988 und 2004 wurden am IVPZ 45557 Sektionsfälle untersucht, wovon 26768 (58.8\%) von landwirtschaftlichen Nutztieren stammten. In diesem Material fanden sich insgesamt 2918 meldepflichtige Tierseuchen, wobei 2426 Nutztiere und 492 andere
Spezies betroffen waren (Tab. 1). Zusätzlich zu den im Ergebnisteil dargestellten Tierseuchen aus dem Sektionsgut wurden bei 4405 Rinderhautbiopsien, welche überwiegend von Kälbern stammten, 492 (11.2\%) BVD positive Fälle gefunden. Die Einteilung in Alterskategorien erfolgte willkürlich. In einigen Fällen war das Alter nicht bekannt. Die Vergleichszahlen des Bundesamtes für Veterinärwesen (BVET) stammen aus der Datenbank des Tierseuchen-Meldesystems zwischen 1991 und 2004 (Tab. 2-5). Die histologische Untersuchung war die weitaus häufigste weiterführende Untersuchung. So wurden beispielsweise im Jahr 2003 84\% aller Autopsien und 90\% der Organuntersuchungen histologisch untersucht. Bakteriologische Abklärungen, als Beispiel einer zusätzlichen Laboruntersuchung, wurden am zweithäufigsten veranlasst (2003:38\% der Autopsien und 75\% der Organeinsendungen). Immunhistochemische Untersuchungen erfolgten, mit Ausnahme der Untersuchung auf BVD, selten. EinVirusnachweis durch Anzüchtung oder eine Polymerase Kettenreaktion (PCR) an Paraffin- oder Frischmaterial erfolgten nur vereinzelt. Bei den 1690 sezierten Nutztieren im Jahr 2003 konnte in 114 Fällen (6.7\%) keine befriedigende Diagnose gestellt werden.

\section{Tierseuchen beim Rind}

Die Untersuchungen von 8849 Rindern beziehungsweise Rinderorganen ergaben insgesamt 1027 meldepflichtige Tierseuchen $(11.6 \%$ der Sektionen bei Rindern) (Tab. 1). Tabelle 2 zeigt die Zahlen der verschiedenen diagnostizierten Tierseuchen. Am häufigsten wurde eine Infektion mit BVD-Virus gefunden. Von den 298 persistent infizierten (PI) Tieren hatten nur 47 Schleimhautläsionen (15.8\%) wie bei einer klinisch erkennbaren Mucosal Disease. Weitere häufig diagnostizierte Tierseuchen waren: Bösartiges Katarrhalfieber (BKF), Bovine Spongiforme Enzephalopathie (BSE), Neosporose und die Listeriose (vorwiegend als ZNS-Manifestation).

Besondere Erwähnung verdienen die Daten beim Abortgeschehen des Rindes zwischen 2000 und 2004. In dieser Zeitspanne wurden insgesamt 372 Aborte und 30 Totgeburten untersucht. Zum Untersuchungsmaterial zählte in 269 Fällen auch die Plazenta, wobei in 99 Fällen eine eitrig-nekrotisierende, eitrige oder nekrotisierende Plazentitis festgestellt wurde, was auf ein

Tabelle 1:Anzahl sezierter Tiere und diagnostizierte Tierseuchen am IVPZ ab 1988.

$\begin{array}{lrrrrrrr} & \text { Rinder } & \text { Schweine } & \text { Schafe } & \text { Ziegen } & \text { Equiden } & \text { Andere } & \Sigma \\ \text { Autopsien } & 4750 & 8254 & 2227 & 999 & 1876 & 18259 & 36365 \\ \text { Organuntersuchungen } & 4099 & 3262 & 545 & 239 & 517 & 530 & 9192 \\ \Sigma & 8849 & 11516 & 2772 & 1238 & 2393 & 18789 & 45557 \\ \text { Tierseuchen } & 1027 & 842 & 335 & 189 & 33 & 492 & 2918\end{array}$


Tabelle 2:Tierseuchen beim Rind am IVPZ aufgeteilt nach Alterskategorien ab 1988*.

\begin{tabular}{|c|c|c|c|c|c|c|c|c|c|c|}
\hline $\begin{array}{l}\text { Alterskategorie } \\
\text { Seuche }\end{array}$ & Aborte & Totgeburten & bis $1 \mathrm{~W}$ & $2-26 \mathrm{~W}$ & $27-51 \mathrm{~W}$ & $1-2 \mathrm{~J}$ & $>2 \mathrm{~J}$ & $\begin{array}{l}\text { Unbe- } \\
\text { kannt }\end{array}$ & $\Sigma$ & $\begin{array}{r}\text { BVET } 1991 \\
-2004 \star \star\end{array}$ \\
\hline $\begin{array}{l}\text { Auszurottende Seuchen } \\
\text { BSE } \\
\text { Tollwut }\end{array}$ & & & & & & & $\begin{array}{r}140 \\
1\end{array}$ & & $\begin{array}{r}140 \\
1\end{array}$ & $\begin{array}{r}454 \\
22\end{array}$ \\
\hline $\begin{array}{l}\text { Zu bekämpfende Seuchen } \\
\text { Salmonellose } \\
\text { Coxiellose } \\
\text { Leptospirose }\end{array}$ & $\begin{array}{l}5 \\
9 \\
1\end{array}$ & 1 & 3 & 9 & & & 12 & $\begin{array}{l}3 \\
1\end{array}$ & $\begin{array}{r}32 \\
10 \\
2\end{array}$ & $\begin{array}{l}827 \\
682 \\
434\end{array}$ \\
\hline $\begin{array}{l}\text { Zu überwachende Seuchen } \\
\text { BVD/MD }^{1} \\
\text { BKF}^{2} \\
\text { Neosporose } \\
\text { Listeriose } \\
\text { Campylobacteriose } \\
\text { Kryptosporidiose } \\
\text { Paratuberkulose } \\
\text { Yersiniose } \\
\text { Chlamydienabort }\end{array}$ & $\begin{array}{r}23 \\
112 \\
10\end{array}$ & 2 & $\begin{array}{r}27 \\
6 \\
1 \\
16 \\
17\end{array}$ & $\begin{array}{r}99 \\
10 \\
10 \\
2 \\
52 \\
20\end{array}$ & $\begin{array}{l}40 \\
10\end{array}$ & $\begin{array}{r}74 \\
40 \\
22 \\
1\end{array}$ & $\begin{array}{r}19 \\
101 \\
\\
59\end{array}$ & $\begin{array}{r}14 \\
13 \\
15 \\
11 \\
2\end{array}$ & $\begin{array}{r}298 \\
174 \\
128 \\
117 \\
80 \\
39 \\
3 \\
2 \\
1\end{array}$ & $\begin{array}{r}343 \\
202 \\
91 \\
91 \\
19 \\
168 \\
70 \\
1 \\
96\end{array}$ \\
\hline$\Sigma$ & 163 & 5 & 70 & 202 & 56 & 137 & 335 & 59 & 1027 & 3500 \\
\hline
\end{tabular}

${ }^{1}$ Bovine Virusdiarrhoe / Mucosal Disease; ${ }^{2}$ Bösartiges Katarrhalfieber

W: Alter in Wochen, J: Alter in Jahren, Unbekannt: Alter nicht bekannt

* Ein Teil der Krankheiten ist erst nach 1988 als Tierseuche deklariert worden

** Gesamtschweizerisch im Tierseuchenmeldesystem publizierte Zahlen von 1991 bis Ende 2004

infektiöses Abortgeschehen hinwies. Das Gehirn wurde von 322 Tieren histologisch untersucht. Die Untersuchung ergab in 54 Fällen Läsionen (kleinherdförmige Nekrosen in Gehirn und/oder Rückenmark sowie unregelmässiges Auftreten von weiteren histologischen Läsionen wie nichteitrige Myokarditis, Myositis oder multifokale Hepatitis), die typisch für eine protozoäre Infektion mit Neospora caninum sind (Rommel, 2000; Dubey, 2003; Dubey et al., 2006). Davon wurde in 29 Fällen die Diagnose immunhistochemisch oder molekularbiologisch (PCR) verifiziert. Aus wissenschaftlichem Interesse wurden knapp 90\% der Aborte immunhistochemisch auf das BVDVirus untersucht, wobei 7 positive Reagenten auftraten. Bakterielle Aborterreger wurden in 27 Fällen $(6.7 \%)$ isoliert, wovon 13 Fälle tierseuchenrelevant waren: Coxiella burnetii (5), Listeria monocytogenes (3), Salmonella spp. (2), Yersinia spp. (2) und Chlamydophila abortus (1) (Pospischil et al., 2002). Weitere bakterielle Erreger in Zusammenhang mit dem Abortgeschehen waren: Arcanobacterium pyogenes (7), Actinomyces spp. (4), E. coli (2) und Histophilus somni (1). Pilzinfektionen wurden in 6 Fällen festgestellt. Bei Rinderaborten wurden somit bei über einem Drittel (137/402) der Fälle Infektionserreger (94) beziehungsweise Hinweise auf ein infektiöses Geschehen (43) festgestellt. Bei den restlichen Fällen waren keine Hinweise auf ein infektiöses Geschehen erkennbar. Die Neosporose beziehungsweise der Verdacht darauf war die weitaus am häufigsten diagnostizierte infektiöse $\mathrm{Ab}$ ortursache und 8-mal häufiger als BVD.

\section{Tierseuchen beim Schwein}

Bei der hohen Anzahl an Organeinsendungen von Schweinen im Alter zwischen 2 und 26 Wochen handelte es sich grösstenteils um Lungen (1466), die im Rahmen der Schlachtkontrolle zur Untersuchung auf die Enzootische Pneumonie beziehungsweise die Aktinobazillose der Schweine eingesandt wurden. Diese Erkrankungen machten allein $91.6 \%$ der diagnostizierten Tierseuchen beim Schwein aus (Tab. 3). Die Diagnosen stützten sich auf einen mikroskopischen Nachweis am Ausstrich von Bronchialschleim und passenden histologischen Läsionen bei EP beziehungsweise auf ein kulturelles Anzüchtungsverfahren mit nachfolgender Erregertypisierung bei APP. Die technischen Weisungen verlangen heute den Nachweis von Mycoplasma hyopneumoniae mittels PCR (BVET, 2005). Die Fälle mit Verdacht auf Teschener Erkrankung bei Mastschweinen basierten auf dem Vorhandensein von histologischen Läsionen und dem Ausschluss von Schweinepest und der Aujeszkyschen Krankheit.

\section{Tierseuchen beim Schaf}

Über ein Drittel (34.3\%) der meldepflichtigen Tierseuchen beim Schaf wurde im Zusammenhang mit 
Tabelle 3:Tierseuchen beim Schwein am IVPZ aufgeteilt nach Alterskategorien ab $1988^{\star}$.

\begin{tabular}{|c|c|c|c|c|c|c|}
\hline $\begin{array}{l}\text { Alterskategorie } \\
\text { Seuche }\end{array}$ & Aborte & $2-26 \mathrm{~W}$ & $>27 \mathrm{~W}$ & $\begin{array}{l}\text { Unbe- } \\
\text { kannt }\end{array}$ & $\Sigma$ & $\begin{array}{r}\text { BVET } 1991 \\
-2004 \star \star\end{array}$ \\
\hline \multicolumn{7}{|l|}{ Zu bekämpfende Seuchen } \\
\hline $\mathrm{EP}^{1}$ & & 550 & 18 & & 568 & 398 \\
\hline $\mathrm{APP}^{2}$ & & 197 & 6 & & 203 & 33 \\
\hline Salmonellose & & 6 & 3 & 2 & 11 & 24 \\
\hline Leptospirose & 1 & & & & 1 & 6 \\
\hline \multicolumn{7}{|c|}{ Zu überwachende Seuchen } \\
\hline Campylobacteriose & & 33 & & 4 & 37 & k.A. \\
\hline Kryptosporidiose & & 5 & & 8 & 13 & k.A. \\
\hline Teschener Krankheit & & 2 & 2 & & 4 & k.A. \\
\hline Echinokokkose & & & 1 & 3 & 4 & k.A. \\
\hline Toxoplasmose & 1 & & & & 1 & k.A. \\
\hline$\Sigma$ & 2 & 793 & 30 & 17 & 842 & 461 \\
\hline
\end{tabular}

${ }^{1}$ Enzootische Pneumonie der Schweine; ${ }^{2}$ Actinobacillose der Schweine

W: Alter in Wochen, J: Alter in Jahren, Unbekannt: Alter nicht bekannt

* Ein Teil der Krankheiten ist erst nach 1988 als Tierseuche deklariert worden

** Gesamtschweizerisch im Tierseuchenmeldesystem publizierte Zahlen von 1991 bis Ende 2004

k. A.: keine Angaben (Vergleichszahlen) vorhanden

Tabelle 4:Tierseuchen beim Schaf am IVPZ aufgeteilt nach Alterskategorien ab 1988*.

\begin{tabular}{|c|c|c|c|c|c|c|c|c|c|}
\hline $\begin{array}{l}\text { Alterskategorie } \\
\text { Seuche }\end{array}$ & Aborte & Totgeburten & bis $1 \mathrm{~W}$ & $2-26 \mathrm{~W}$ & $27-51 \mathrm{~W}$ & $>1 \mathrm{~J}$ & $\begin{array}{l}\text { Unbe- } \\
\text { kannt }\end{array}$ & $\Sigma$ & $\begin{array}{r}\text { BVET } 1991 \\
-2004 \star \star\end{array}$ \\
\hline $\begin{array}{l}\text { Auszurottende Seuchen } \\
\text { Traberkrankheit }\end{array}$ & & & & & & 4 & & 4 & 9 \\
\hline $\begin{array}{l}\text { Zu bekämpfende Seuchen } \\
\text { Salmonellose } \\
\text { Brucellose }^{1} \\
\text { Coxiellose }\end{array}$ & $\begin{array}{l}1 \\
1 \\
2\end{array}$ & & & 3 & & 4 & $\begin{array}{l}3 \\
1\end{array}$ & $\begin{array}{r}14 \\
3 \\
2\end{array}$ & $\begin{array}{r}25 \\
101 \\
35\end{array}$ \\
\hline Zu überwachende Seuchen & & & & & & & & & \\
\hline $\begin{array}{l}\text { Listeriose } \\
\text { Chlamydiose }^{2}\end{array}$ & $\begin{array}{r}1 \\
73\end{array}$ & $\begin{array}{l}1 \\
7\end{array}$ & 11 & 40 & 6 & 65 & 20 & $\begin{array}{r}133 \\
91\end{array}$ & $\begin{array}{r}71 \\
278\end{array}$ \\
\hline Toxoplasmose & 23 & 2 & 5 & 2 & & 1 & 1 & 34 & 6 \\
\hline Lungenadenomatose & & & & & & 13 & 9 & 22 & 14 \\
\hline Maedi-Visna & & & & & & 9 & 3 & 12 & 56 \\
\hline Pseudotuberkulose ${ }^{2}$ & 1 & & & 1 & & 1 & 5 & 8 & 8 \\
\hline Campylobacteriose & 1 & & 2 & 3 & & & 1 & 7 & 2 \\
\hline Paratuberkulose & & & & & & 3 & 2 & 5 & 3 \\
\hline Neosporose & 1 & & 2 & & & & & 3 & k.A. \\
\hline Yersiniose & 1 & & & & & & & 1 & k.A. \\
\hline$\Sigma$ & 105 & 10 & 20 & 49 & 6 & 100 & 45 & 335 & 709 \\
\hline
\end{tabular}

${ }^{1}$ der Widder; ${ }^{2}$ der Schafe und Ziegen

W: Alter in Wochen, J: Alter in Jahren, Unbekannt: Alter nicht bekannt

* Ein Teil der Krankheiten ist erst nach 1988 als Tierseuche deklariert worden

** Gesamtschweizerisch im Tierseuchenmeldesystem publizierte Zahlen von 1991 bis Ende 2004

k.A.: keine Angaben (Vergleichszahlen) vorhanden

Aborten und Totgeburten diagnostiziert (Tab. 4). Der Chlamydienabort war mit grossem Abstand die häufigste Abortursache beim Schaf und konnte auch mit Lebensschwäche neugeborener Lämmer (Alter <1 Woche) in Zusammenhang gebracht werden. Der Nachweis des Erregers erfolgte ausschliesslich über Immunhistochemie (IHC) (primärer Antikörper: cLPS clone AC-1, Progen, Heidelberg, Deutschland).
Der Erregernachweis der zweithäufigsten Abortursache in unserem Sektionsgut, der Toxoplasmose, erfolgte 29-mal mittels IHC (primärer Antikörper:Toxoplasma gondii Ab1, Lab Vision, Warm Springs, USA), und zweimal mittels PCR. In 3 Fällen waren typische Läsionen vorhanden, aber ohne erfolgreichen Erregernachweis. Drei Infektionen mit Neospora caninum konnten zweifelsfrei mittels IHC nachgewie- 
sen werden. Listeria monocytogenes Infektionen, die am häufigsten diagnostizierte Tierseuche beim Schaf, verliefen vorwiegend als zerebrale Erkrankung. Das jüngste Lamm mit Gehirnlisteriose war laut Anamnese knapp 1 Monat alt. Der Grossteil der Diagnosen wurde histomorphologisch anhand des pathognostischen Bildes gestellt.

Die Diagnosen Adenomatose und Maedi-Visna wurden ausschliesslich auf Grund der histologischen Lungenläsionen diagnostiziert.Von den 4 histologisch diagnostizierten Scrapiefällen zeigte mindestens ein Fall die atypische Form (Ehrensperger et al., 2004).

\section{Tierseuchen bei der Ziege}

Mehr als die Hälfte (51.3\%) der bei der Ziege diagnostizierten Tierseuchen betrafen Abortfälle (Tab. 5). Die drei häufigsten Aborterreger waren, wie beim Schaf, Chlamydophila abortus, Toxoplasma gondii und Coxiella burnetii. Auch bei Ziegenaborten wurde einmal eine Infektion mit Neospora caninum nachgewiesen. Die jüngste Ziege mit Gehirnlisteriose war ebenfalls, wie beim Schaf, gemäss Anamnese, knapp 1 Monat alt. Mit zehn Wochen wurde bei der jüngsten von vier Ziegen Caprine Arthritis Enzephalitis (CAE) anhand der histologischen Gehirnläsionen diagnostiziert. Bei den weiteren anhand histologischer Veränderungen gestellten Verdachtsdiagnosen auf CAE zeigten zwei Tiere Läsionen im ZNS und je einmal Gelenks- oder Lungenveränderungen, zwei Fälle wiesen nur Gelenksveränderungen auf.

\section{Tierseuchen bei den Equiden}

Bei Equiden fanden sich lediglich 33 Fälle von meldepflichtigen Tierseuchen, dabei handelte es sich in 8 Fällen um Listeriosen darunter 5 septikämische Fälle bei 3 neonatalen Fohlen, einem etwas älteren Fohlen und einem 18-jährigen Esel. Drei erwachsene Pferde erkrankten an der beim Pferd seltenen, zerebralen Listeriose. In diesen drei Fällen konnte in zwei Fällen Listerienantigen mittels IHC im Gehirn nachgewiesen werden, in einem Fall gelang auch der Speziesnachweis (Listeria monocytogenes) mittels Fluoreszenz in situ Hybridisierung (FISH) (Rütten et al., 2006). In 15 Fällen wurden Salmonella spp. isoliert, in 12 Fällen handelte es sich um erwachsene Tiere. Echinococcus granulosus Finnen konnten in 10 Fällen bei alten Pferden (14-36 Jahre) diagnostiziert werden.

\section{Tierseuchen anderer Spezies}

Die Ergebnisse beschränken sich auf die häufigsten und auf einige besondere Beobachtungen bei insgesamt 492 Fällen von Tierseuchen (2.6\% der Sektionsfälle) bei anderen Tierspezies. Bei 40 Hunden konnten als meldepflichtige Erreger in jeweils 12 Fällen Salmonella spp. und Campylobacter spp. isoliert werden. Bei 6 Hunden wurde Echinococcus granulosus gefunden, wobei in 4 Fällen eine Organmanifestation und in 2 Fällen ein Darmbefall festgestellt werden konnte. Infektionen mit Neospora caninum traten bei 8 Hunden auf, wobei 4 Welpen im Alter zwischen 1 und 6 Wo-

Tabelle 5:Tierseuchen bei der Ziege am IVPZ aufgeteilt nach Alterskategorien ab 1988*

\begin{tabular}{|c|c|c|c|c|c|c|c|c|c|}
\hline $\begin{array}{l}\text { Alterskategorie } \\
\text { Seuche }\end{array}$ & Aborte & Totgeburten & bis $1 \mathrm{~W}$ & $2-26 \mathrm{~W}$ & $27-51 W$ & $>1 \mathrm{~J}$ & $\begin{array}{l}\text { Unbe- } \\
\text { kannt }\end{array}$ & $\Sigma$ & $\begin{array}{r}\text { BVET } 1991 \\
-2004 \star \star\end{array}$ \\
\hline $\begin{array}{l}\text { Auszurottende Seuchen } \\
\text { CAE }^{1}\end{array}$ & & & & 4 & & 5 & 1 & 10 & 1095 \\
\hline $\begin{array}{l}\text { Zu bekämpfende Seuchen } \\
\text { Coxiellose } \\
\text { Salmonellose }\end{array}$ & 18 & & 1 & & & & & $\begin{array}{r}18 \\
1\end{array}$ & $\begin{array}{r}42 \\
6\end{array}$ \\
\hline Zu überwachende Seuchen & & & & & & & & & \\
\hline $\begin{array}{l}\text { Listeriose } \\
\text { Chlamydiose }^{2}\end{array}$ & $\begin{array}{r}7 \\
46\end{array}$ & 2 & $\begin{array}{l}1 \\
1\end{array}$ & 3 & 2 & 41 & 13 & $\begin{array}{l}67 \\
49\end{array}$ & $\begin{array}{r}50 \\
120\end{array}$ \\
\hline Toxoplasmose & 22 & & 1 & & & & & 23 & 3 \\
\hline Paratuberkulose & & & & & & 8 & 2 & 10 & 5 \\
\hline Kryptosporidiose & & & 1 & 2 & & & 2 & 5 & k.A. \\
\hline Campylobacteriose & & & 1 & 3 & & & & 4 & k.A. \\
\hline Neosporose & 1 & & & & & & & 1 & k.A. \\
\hline Yersiniose & 1 & & & & & & & 1 & k.A. \\
\hline$\Sigma$ & 95 & 2 & 6 & 12 & 2 & 54 & 18 & 189 & 1321 \\
\hline
\end{tabular}

${ }^{1}$ Caprine Arthritis-Encephalitis; ${ }^{2}$ der Schafe und Ziegen

W: Alter in Wochen, J: Alter in Jahren, Unbekannt: Alter nicht bekannt

* Ein Teil der Krankheiten ist erst nach 1988 als Tierseuche deklariert worden

** Gesamtschweizerisch im Tierseuchenmeldesystem publizierte Zahlen von 1991 bis Ende 2004

k.A.: keine Angaben (Vergleichszahlen) vorhanden 
chen einen Befall des ZNS zeigten und in einem Fall zusätzlich die Leber betroffen war. Bei einem 4 Wochen alten Welpen war in der Herzmuskulatur Neospora-Antigen immunhistochemisch nachweisbar. Bei 4 Hunden zwischen 20 Wochen und 2.5 Jahren war jeweils im ZNS Neospora-Antigen nachweisbar. In einem Fall wurde das Genom des Erregers mittels PCR nachgewiesen. Im Jahre 1993 wurde bei einem 16 Wochen alten Hund eine Tollwutinfektion diagnostiziert.

Tierseuchen oder Zoonosen bei Katzen fanden sich insgesamt bei 59 Patienten. Darunter waren 30 Fälle von Toxoplasmose: 17-mal mit ZNS-Manifestation und 11-mal mit Ausbreitung in verschiedene Organe. Nur in 2 dieser Fälle wurde die Darminfektion festgestellt. Bei 16 Katzen konnten Salmonella spp. gefunden werden, Campylobacter spp. wurden bei 5 Tieren isoliert. In den Jahren 1996 und 2003 gelang der Nachweis der Transmissiblen Spongiformen Enzephalopathie (TSE) bei 2 Katzen (Soldati et al., 2004). Zwei weitere exotische Erkrankungen traten als septikämische Form der Listeriose und als Tuberkulose (Lungenform mit Beteiligung diverser Lymphknoten) auf. Im letzteren Fall wurde die Anwesenheit von Mykobakterien des Mycobacterium tuberculosisKomplexes mittels Gensondentechnik (PCR) verifiziert.

Nagetiere erkrankten häufig (101 Fälle) an Yersiniose (Yersinia pseudotuberculosis). Bei Reptilien, insbesondere bei Schlangen, wurden in 196 Fällen Salomonella spp. festgestellt. Dabei handelte es sich fast ausschliesslich um den bei Reptilien häufigen Darmbefall ohne assoziierte histologische Läsion (Frye, 1991). Im Jahre 1988 wurde bei einem 24-jährigen Indischen Elefanten aus dem Zürcher Zoo Tuberkulose (Mycobacterium tuberculosis) durch die Typisierung der lichtmikroskopisch säurefesten Stäbchen diagnostiziert. Wir konnten auch bei einzelnen Hirschen aus Wildgehegen und zoologischen Gärten BKF, Pseudotuberkulose, Kryptosporidien (2 Junghirsche), Paratuberkulose, Listeriose und Salmonellose nachweisen. Elf Primaten aus zoologischen Gärten hatten Leber- oder Lungeninfektionen mit Echinokokken, wobei es sich mehrheitlich um Infektionen mit Echinococcus multilocularis gehandelt hat.

\section{Diskussion}

Die vorgelegten Daten sprechen dafür, dass ein Universitätsinstitut für Veterinärpathologie zusammen mit ihren Unterauftragnehmern bei gewissen Tierseuchen mittels des pathologisch-anatomischen Untersuchungsganges und gegebenenfalls spezifischen Zusatzuntersuchungen einen vergleichsweise hohen Anteil der Tierseuchen aufdecken kann. Tierseuchen oder Seuchenverdachtsfälle wurden in dieser Unter- suchung bei $9.1 \%$ aller Nutztiere festgestellt. Die Wahrscheinlichkeit, dass wesentliche apparente Tierseuchen im Rahmen eines pathologisch-anatomischen Untersuchungsganges übersehen werden, wird durch weitere Kontrollmechanismen klein gehalten. Die Interpretation aller Untersuchungen erlaubt in den meisten Fällen eine endgültige Beurteilung des Falles (Bayerisches LGL, 2007). Kann die Todesursache durch eine konkrete Krankheit erklärt werden, ist das zusätzlicheVorhandensein einer Tierseuche wenig wahrscheinlich. Im schon erwähnten Jahr 2003 wurde in unserem Sektionsgut bei 1630 sezierten Nutztieren nur in 114 Fällen (6.7\%) keine befriedigenden Diagnosen gestellt; davon waren rund die Hälfte (55) Abortfälle ohne Hinweise auf eine Ätiologie.Tierseuchen oder derVerdacht darauf müssen den Behörden schriftlich und ohne Verzug gemeldet werden. Bei hoch ansteckenden, auszurottenden und zu bekämpfenden Tierseuchen werden die zuständigen Veterinärämter sofort telefonisch orientiert. Das BVET unterhält mit dem Tierseuchenmeldesystem der Schweiz seit einigen Jahren eine zentrale Datenbank über diagnostizierte Tierseuchen. Eine direkte Meldung durch die akkreditierten Untersuchungslaboratorien auch dorthin wird erst neuerdings durchgefuihrt (Art. 312, TSV).

Vergleicht man die Anzahl diagnostizierter Tierseuchen aus unserem Institut mit den Zahlen des Tierseuchenmeldesystems (Tab. 2-5), machen unsere Zahlen bei diversen Tierseuchen einen beachtlichen Anteil aus und übersteigen teilweise sogar die Zahlen der Datenbank des Tierseuchenmeldesystems. Zum Teil deutlich höhere Tierseuchenzahlen in unserem Untersuchungsgut (Tab. 2-5) waren beim Toxoplasmenabort der kleinen Wiederkäuer (mehr als 6-mal soviel), der Paratuberkulose der kleinen Wiederkäuer, der Lungenadenomatose, den Listeriosefällen aller Wiederkäuer und der Neosporose der Rinder zu beobachten. Für diese Abweichungen gibt es mehrere Erklärungen. Die Datenbank des Tierseuchenmeldesystems reicht nur bis 1991 zurück. Einige Tierseuchen wie EP und APP und alle zu überwachenden Tierseuchen sind dies erst seit neuerem Datum; unser Auswertungsprogramm zählte aber alle diese Krankheiten als Tierseuchen ab 1988. Des Weiteren scheint die Weitergabe der schriftlichen Meldungen an das BVET beziehungsweise der Eintrag in die Tierseuchendatenbank nicht immer erfolgt zu sein. Hohe Prävalenzen gewisser Tierseuchen im Sektionsgut lassen sich dadurch erklären, dass ein Sektionsgut nie die realen Häufigkeiten widerspiegelt, da das Einsendegut vorselektiert ist. Auch die diagnostische Kompetenz oder Forschungsinteressen des Untersuchungsinstituts und ein damit verbundener möglicher Preisnachlass spielen eine Rolle. Das IVPZ ist nicht nur Referenzlabor für den Chlamydienabort der Schafe und Ziegen, sondern bemühte sich auch aus wissenschaft- 
lichen Gründen um diese Aborte, was die hohen Zahlen an positiven Chlamydienproben (Chlamydophila abortus) im Sektionsgut erklärt. Die Diagnose einer Tierseuche beziehungsweise derVerdacht darauf kann auf sehr unterschiedlichen diagnostischen Vorgehen beruhen: dem Nachweis von Antikörpern gegen einen bestimmten Erreger, der Erregeranzüchtung, dem Erregernachweis mittels molekularbiologischer Techniken (PCR), dem Vorhandensein von pathognostischen Läsionen (nur bei wenigen Krankheiten) oder demVorhandensein von Läsionen, die bestimmte Tierseuchen in die Differentialdiagnosen mit einschliessen. Je nach Erreger müssen die Resultate unterschiedlicher diagnostischer Verfahren sehr sorgfältig interpretiert werden, insbesondere serologische Ergebnisse.

Einige andere interessante Feststellungen sollen hier ebenfalls zur Diskussion kommen. Nur wenige Tierseuchen zeigen pathognostische histologische Läsionen wie zum Beispiel die zerebrale Form der Listeriose. Auf den Erregernachweis wird bei der Listeriose in der Regel verzichtet, weil die Histologie aus unserer Sicht sensitiver ist als der bakteriologische Nachweis (Ehrensperger et al., 2001). Parasitäre Infektionen, verursacht durch Protozoen vom Stamm Apicomplexa, wie Toxoplasma gondii, Neospora caninum und beim Rind differentialdiagnostisch auch Sarcocystis cruzi zeigen histomorphologisch ebenfalls eine typische Charakteristik (Rommel, 2000). Insbesondere für N. caninum Infektionen bestehen bei abortierten Feten die häufigsten Läsionen aus einer multifokalen Enzephalitis und/oder multifokalen nichteitrigen Myositis und Myokarditis (Dubey, 2003; Dubey et al., 2006; Foster, 2007). Nur auf histomorphologischer Basis alleine kann allerdings nicht zwischen den Erregern Toxoplasma und Neospora unterschieden werden. Hierfür sind IHC- oder PCR-Techniken unumgänglich. Die wenigen Berichte über mit $T$. gondii infizierten Rinderfeten (Sager et al., 2001; Canada et al., 2002) zeigen, dass die Bedeutung von T. gondii beim Abortgeschehen des Rindes als gering einzustufen ist. Beim kleinen Wiederkäuer zeigt sich ein umgekehrtes Bild; hier ist Toxoplasma gondii weitaus häufiger als Neospora caninum (Dubey, 2003).

Intrauterine Infektionen mit Neospora caninum können auch erst in den ersten Lebenswochen zu Symptomen führen. Die berichteten Fälle von Kälbern, die ZNS-Symptome entwickelten, waren alle jünger als 4 Monate (Dubey und Schares, 2006). In unserem Material war das älteste Kalb mit Verdacht auf Neosporose 8 Wochen, das älteste mit durch IHC bewiesenem Erregernachweis 2 Wochen alt. Die meisten der geborenen Kälber mit der Diagnose oder Verdachtsdiagnose Neosporose zeigten Symptome wie Festliegen seit Geburt, Ataxie, Astasie, Hinterhandschwäche und Muskelatrophie. Die postpartale Neosporose mit ZNS-Symptomatik ist also eine Differentialdiagnose zur Spinalen Muskelatrophie (SMA), Spinalen Dysmyelinisierung (SDM) und anderen Krankheiten, die zum Festliegen führen. T. gondii ist ein wichtiger Zoonoseerreger für den Menschen, für N. caninum gibt es keine Hinweise auf ein eventuelles zoonotisches Potential (Dubey et al., 2006).

Über den Untersuchungszeitraum hinweg hat sich die Diagnostik bei diversen Erkrankungen verändert. So war früher die Histopathologie das Verfahren der Wahl, um BKF definitiv zu diagnostizieren; heute kann die Diagnose schon am lebenden Tier mittels PCR-Verfahren gestellt werden (Müller-Doblies et al., 1998). Auch die Transmissiblen Spongiformen Enzephalopathien waren bis etwa 1996 ausschliesslich anhand der histopathologischen Läsionen diagnostizierbar. Erst danach kamen die Schnelltests (Western Blot und ELISA) und die IHC dazu.

Das häufige Trägertum von Salmonellen bei Reptilien - nach Literatur etwa bei 50-90\% der Tiere (Chiodini and Sundberg, 1981) - wird hinsichtlich Bedeutung und zoonotischer Gefahr unterschiedlich beurteilt. In Schweden sind Zahlen über die Häufigkeiten von Salmonellenerkrankungen beim Menschen, die zu Salmonelleninfektionen bei Reptilien assoziiert waren, bekannt. Die Inzidenz von 0.15 Erkrankungsfällen beim Menschen auf 100000 Einwohner nahm nach dem EU Beitritt und der Importmöglichkeit von nicht Salmonellen freien Reptilien auf 0.79 Fälle/100 $000 \mathrm{zu}$ (De Jong et al., 2005). Bei Kontakt mit Reptilien oder deren Lebensraum sind hygienische Aspekte verstärkt zu beachten. Eine Aufklärungskampagne in Schweden liess die Inzidenz von 0.79 wieder auf 0.46/100 000 sinken (De Jong et al., 2005).

Der Nachweis einer persistenten Infektion mit dem BVD Virus (PI Tiere) bei Einzeltieren wurde am IVPZ bis heute mittels IHC an unfixiertem oder fixiertem Gewebe durchgeführt. Der hohe Anteil BVD positiver Reagenten beim Kalb (11.2\%), der deutlich höher ist als bei Erhebungen auf Bestandesebene mit 7.7\% (Siegwart et al., 2005) beziehungsweise auf Alpen erhobenen Daten mit 0.9\% (Schönmann, 1997) respektive mit 0.1\% (Landolt, 1995) Prävalenz, ist mit grosser Wahrscheinlichkeit Folge einer Vorselektion von PI verdächtigen Tieren durch die praktizierenden Tierärzte.

\section{Dank}

Diese Arbeit wurde vom Bundesamt fürVeterinärwesen finanziert (Projektnummer 1.04.06). 


\section{Epizooties à déclaration obligatoire diagnosti- quées à l'Institut de pathologie vétérinaire de I'Université de Zurich entre 1988 et 2004}

L'examen et la documentation des lésions macroscopiques et histologiques sur les cadavres d'animaux sont l'une des tâches centrales de la pathologie vétérinaire. Il arrive fréquemment que l'expéditeur demande des éclaircissements sur la maladie respectivement la cause de la mort sans donner une anamnèse précise ou sans poser de questions spécifiques par exemple quant à la présence d'une épizootie. L'examen consiste en une autopsie selon un schéma habituel et une palette variable d'examens complémentaires pour permettre d'éclaircir le cas et de répondre aux questions de façon économiquement acceptable. Le but de l'analyse rétrospective des autopsies de 36365 cadavres et de 9192 organes entre 1988 et 2004 était de définir le nombre et le type d'épizootie dans le cadre des diagnostiques de routine. Dans 2918 cas (6,4 \% de tous les examens), on était en présence d'épizootie à déclaration obligatoire soit chez 2426 animaux de rente $(9,1 \%$ des animaux de rente) et 492 animaux d'autres espèces $(2,5 \%$ de ces animaux) Ces informations démontrent l'utilité indiscutable des examens anatomopathologiques et permettent de compléter les banques de données existantes.

\section{Literatur}

Amtsblatt der Europäischen Gemeinschaften C 32444 Jahrgang, 20. November 2001.

Bayerisches Landesamt für Gesundheit und Lebensmittelsicherheit, 2007; URL: http://www.lgl.bayern.de/veterinaer/pathologie.htm.

BVET,Bundesamt fürVeterinärwesen:Übersicht Krankheiten. URL: http://www.bvet.admin.ch/gesundheit_tiere/ 01065/index.html?lang=de, 12.01.2007

BVET, Bundesamt fürVeterinärwesen:Technische Weisungen über die Entnahme von Proben und deren Untersuchung auf die Enzootische Penumonie der Schweine (EP), URL: http//www.bvet.admin.ch/gesundheit_tiere/01551/ index.html?lang=de, 2005

BVET, Bundesamt für Veterinärwesen: Tierseuchenmeldesystem der Schweiz. URL: http://www.bvet.admin.ch/ tsmd/, 2007.

Canada N., Meireles C. S., Rocha A., Correia da Costa J. M., Erickson M. W., Dubey J. P.: Isolation of viable Toxoplasma gondii from naturally-infected aborted bovine fetuses. J. Parasitol. 2002, 88: 1247-1248.

De Jong B., Andersson Y., Ekdahl K.: Effect of regulation and education on reptile-associated salmonellosis. Emerg. Infect. Dis. 2005, 11: 398-403.

\section{Epizoozie con obbligo di dichiarazione diag- nosticate all'Istituto di patologia veterinaria dell'Università di Zurigo tra il 1988 e il 2004}

Le analisi e il rilevamento di lesioni macroscopiche ed istologiche nei cadaveri di animali sono uno dei compiti centrali della patologia veterinaria. Spesso i mandanti di necroscopie richiedono dei chiarimenti sulla causa della malattia o della morte senza trasmettere una anamnesi precisa o porre domande specifiche ad esempio sulla presenza di un'epizoozia. L'esame si articola in una necroscopia svolta secondo uno schema generale e in un ventaglio flessibile di esami supplementari necessari a chiarire il caso e ad evadere le domande del mandante; costi e sforzi devono peraltro essere mantenuti entro limiti sostenibili. Scopo di questa analisi retrospettiva di un fondo di necroscopie di 36365 corpi e di 9192 organi di animali operate tra il 1988 e il 2004 era di chiarire il numero e il tipo di epizoozie identificate nell'ambito della diagnostica di routine. Sono state trovate epizoozie con obbligo di dichiarazione in 2918 casi (6.4\% di tutti i casi) comprendenti 2426 animali da reddito $(9.1 \%$ degli animali da reddito) e 492 animali di altre specie $(2.6 \%$ di questo gruppo). Questi dati dimostrano il notevole valore dei chiarimenti anatomico-patologici di routine e possono essere usati per completare le banche dati esistenti.

Dubey J. P.: Review of Neospora caninum and neosporosis in animals. Kor. J. Parasitol. 2003, 41,1: 1-16.

Dubey J. P., Buxton D., Wouda W.: Pathogenesis of bovine neosporosis. J. Comp. Path. 2006, 134: 267-289.

Dubey, J. P., Schares G. : Diagnosis of bovine neosporosis.Vet. Parasit. 2006, 140: 1-34.

Ehrensperger F, Hilbe M., Sydler T., Corboz L., Braun U., Pospischil $A$.: Zerebrale Listeriose bei Schaf und Ziege: eine histopathologische und immunhistologische Studie. Wien. Tierärztl. Mschr. 2001, 88: 219-225.

Ehrensperger F., Soldati G., Hilbe M., Zlinsky K., Raeber A. Schiller I., Botteron C., Siegwart N.: Atypical Scrapie in Switzerland: Case Report with Diagnostic and Epidemiologic Relevance. 22nd Meeting of the European Society of Veterinary Pathology, Olsztyn, Poland, 15-18 September 2004.

Europäische Union:Tiergesundheit und Tierschutz. URL: http://europa.eu.int/comm/food/animal/index_de.htm.

Foster R. A.: Female reproductive system. In: Pathologic Basis of Veterinary Disease.Ed. M. D. McGavin 2007, 18:1300.

Frye F. L.: Infectious Diseases, Infections due to Salmonella and Arizona. In:Biomedical and Surgical Aspects of Captive Reptile Husbandry. Ed. F. L., Frye 1991, 4: 128-129. 
Hilbe M., Ossent P., Zlinksky K., Ehrensperger F: Abnormal bone development associated with BovineVirus Diarrhoea Virus (BVDV) infection in a newborn calf. Europ. J. Vet. Path. 2000, 6: 115-119.

Landolt G.: Epidemiologische Untersuchungen über das Vorkommen von MD-VD beim Rind. Dissertation, Universität Zürich, 1995.

Mayr A.: Gesetzliche Grundlagen derTierseuchenbekämpfung. In: Medizinische Mikrobiologie, Infektions und Seuchenlehre. Hrsg. A. Mayr. Enke Verlag, Stuttgart. 2002, 633-640.

Müller-Doblies U. U, Li H., Hauser B., Adler H., Ackermann $M$. : Field validation of laboratory tests for clinical diagnosis of sheep-associated malignant catarrhal fever.J. Clin. Microbiol. 1998, 2970-2972.

OIE Objectives: URL: http://www.oie.int/eng/OIE/en_ objectifs.htm, 2007.

OIE Old Classification List A: URL: http://www.oie.int/ eng/maladies/en_OldClassification.htm\#ListeA, 2005.

Pospischil A., Thoma R., von BomhardW., Reitt K., Cantieni J., Zimmermann D., Polkinghorne A.: Abort beim Rind durch Chlamydia psittaci. Schweiz. Arch. Tierheilk. 2002, 144: 467-472.

Rommel M.: Protozoeninfektionen derWiederkäuer. In:Veterinärmedizinische Parasitologie. Hrsg. M. Rommel, J. Eckert, E. Kutzer, W. Körting, T. Schnieder. 5. Aufl. Parey, Berlin, 2000, 121-188.
Rütten M., Lehner A., Pospischil A., Sydler T.: Cerebral Listeriosis in an Adult Freiberger Gelding. J. Comp. Path. 2006, 134: 249-253.

Sager H., Fischer I., Furrer K., Strasser M., Waldvogel A., Boerlin P., Audigé L., Gottstein B. : A Swiss case-control study to assess Neospora caninum-associated bovine abortions by PCR, histopathology and serology.Vet. Parasitol. 2001, 102: $1-15$.

Schönmann M.: Epidemiologische Untersuchungen über die Verbreitung der BVD-Virusinfektion auf schweizerischen Rinderalpen. Dissertation, Universität Zürich, 1997.

Siegwart N., Hilbe M., Hässig M., Braun U.: Increased risk of BVDV infection of calves from pregnant dams on communal Alpine pastures in Switzerland. Vet. J. 2006, 172: 386-388.

Soldati G., Hilbe M., Zlinsky K., Schiller I., Ehrensperger F: Immunohistochemical study of PrPSC in neural, lymphoid and other peripheral tissues of a cat with Feline Spongiform Encephalopathy. Abstracts from 22nd meeting of the European Society of Veterinary Pathology, Olysztyn, Poland. 2004, p 205.

Tierseuchengesetz (TSG) vom 1. Juli 1966 (Stand am 13. Juni 2006). Die Bundesbehörden der Schweizerischen Eidgenossenschaft.

Tierseuchenverordnung (TSV) vom 27. Juni 1995 (Stand am 20. Dezember 2005). Die Bundesbehörden der Schweizerischen Eidgenossenschaft.

\section{Korrespondenzadresse}

Dr. Titus Sydler, Institut für Veterinärpathologie, Universität Zürich, Winterthurerstrasse 268, CH-8057 Zürich.E-Mail: tsyd@vetpath.uzh.ch

Manuskripteingang: 17. März 2007

Angenommen: 4. September 2007 